\title{
RADIOCHROMIC FILM CALIBRATION FOR DOSIMETRY IN CHEST CT SCANS
}

\author{
Gómez $^{a}$ A. M. L. and Mourão ${ }^{a}$ A. P. \\ ${ }^{a}$ Departamento de Engenharia Nuclear - Escola de Engenharia Universidade Federal de Minas Gerais \\ Av. Antônio Carlos, 6627. CEP 31270-90 \\ amlgphys@gmail.com
}

\begin{abstract}
Phantoms can be used for image quality control in computed tomography (CT) and dosimetry in specific regions of interest. CT phantoms can be used to modify the parameters of acquisition protocol in order to reduce doses in patients while maintaining the diagnostic image quality. Radiochromic films, GAFCHROMIC XR-AQ2 model, are used to measure absorbed dose responses when exposed to radiation. For the measurement of absorbed dose inside phantoms, it is necessary to not the absorbed dose range to be measured, in order to correlate the darkening of the film with the absorbed dose values. The calibration curves were obtained from radiochromic films using a cylindrical phantom, made of polymethylmethacrylate (PMMA). This phantom has five openings, one central and four peripherals lagged $90{ }^{\circ}$. The peripherals openings were identified of 3, 6, 9 and 12 as an analog clock position hour. The CT phantom was placed in the isocenter of the gantry of the Toshiba scanner, model Asterion. The peripheral openings were used to aligned the phantom with horizontal and vertical lasers. The phantom was irradiated with the voltages in the X-ray tube of 120 and $135 \mathrm{kV}$. To record Air Kerma in PMMA values, a pencil ionization chamber was inserted in the central and 12 openings and the central slice of the phantom was irradiated, in the axial mode. The other openings were filled with PMMA rods. Strips of radiochromic film were placed in special PMMA rods and the central and 12 openings were filled. After the irradiation, images of the radiochromic film strips were obtained and processed in order to have the calibration curves.
\end{abstract}

Keywords: Computed Tomography, Radiochromic film, Calibration curves. 


\section{INTRODUCTION}

The versatility and speed with which diagnostic images are acquired by computed tomography (CT) have influenced the increase in the frequency of such tests for different regions of the patient's body. A single CT test does not represent a significant risk for induction in the development of lesions or tumor tissue. However, the increasing of approved procedures in the Unified Health System (SUS) in Brazil means a high probability of impact on the public health [1-3].

The development of techniques to optimize the processes of acquiring images is very important in the adjustment of the acquisition parameters, the use of iterative image acquisition methods, current automatic control, etc. to seek an absorbed dose reduction in patients and maintaining image quality. Dosimetric studies in patients generate results that show the punctual absorbed dose in specific regions highly sensitive to ionizing radiation [4-6].

The phantoms are used in CT scans for image quality control, calibration and dosimetry. Even is not possible to reproduce in detail the human body characteristics, them allow to keep control of the average absorbed dose in measurements made by detectors. Radiochromic films are able to detect ionizing radiation coming from different directions and thus obtain maps of air Kerma profiles in a proportional response to their darkening $[7,8]$.

Calibration curves of radiochromic films are important to convert the film darkening by irradiation process in absorbed doses. Thus, it is possible to measure specific doses in CT scanning processes by using phantoms of different shapes and compositions to propose optimized acquisition protocols.

\section{MATERIALS AND METHODS}

The standard cylindrical chest phantom was used to measure Air Kerma in PMMA. The PMMA phantom is $32 \mathrm{~cm}$ in diameter and $15 \mathrm{~cm}$ long. This phantom has five openings, one central and 
four peripherals of $1.12 \mathrm{~cm}$ in diameter and $15 \mathrm{~cm}$ long. The peripheral openings center is $1 \mathrm{~cm}$ from the surface of the cylindrical phantom. Peripheral openings are lagged $90^{\circ}$.

Radiochromic film strips of $2 \times 0.5 \mathrm{~cm}^{2}$ were cut from a GAFCHROMIC XR-AQ2 sheet. They were used to record absorbed dose of an X-ray beam in CT scan expositions. The sensitivity to radiation is between 0.1 and $20 \mathrm{cGy}$ to X-ray beams [9]. The pencil ionization chamber, model ACCU-RADCAL GOLD 10X6-3 measures longitudinal Air Kerma values in CT X-ray beams. This ionization chamber can recorder air Kerma values in a range of $200 \mathrm{nGy}$ to $1 \mathrm{kGy}$ [10].

The tests were done in a Toshiba CT scanner with 4 channels, Aston model. The PMMA chest phantom was positioned in the isocenter of the gantry with the help of lasers. The peripheral openings were aligned on a vertical and horizontal axis, and they were called 3, 6, 9 and 12 as the hours of an analog clock. The radiochromic film strips were placed into PMMA rods filling the openings in the position 12 and central. Solid PMMA rods were inserted into other openings. The helical CT scans irradiated a distance of $10 \mathrm{~cm}$ in the central region of the phantom, with voltages of 120 and $135 \mathrm{kV}$. For the $120 \mathrm{kV}$ beam, the film strips were irradiated in four steps, with 50, 100, 200, and $300 \mathrm{~mA} . \mathrm{s}$, and for the $135 \mathrm{kV}$ beam, they were irradiated in three steps, with 50, 100 and 200 mA.s

The ionization chamber was placed in the "central" and " 12 " openings of the phantom to measure of Air Kerma in PMMA. The central slice of the phantom with $10 \mathrm{~mm}$ thickness was irradiated in axial mode. Five measurements were done in each position for voltages of 120 and 135 $\mathrm{kV}$ and 50, 100, 200, and $300 \mathrm{~mA} . \mathrm{s}$.

After 24 hours of the film strips exposition it was obtained digital images of them in .JPG format at a resolution of $300 \mathrm{ppi}$. Images were processed using ImageJ software. The color image of the strips in RGB was split and the red channel image was used to obtain the responses. The red channel presents the better intensity variation. The red channel images of the exposed and unexposed strips had the greyscale inverted. The intensity values in greyscale to unexposed films were treated as Background (BG) and considered as zero miligray. 


\section{RESULTS}

Two calibration curves were obtained, one for each voltage of the X-ray tube, because radio chromic films have energy dependency. These curves allow the conversion of the dark signal recorded by the film strips in air Kerma in PMMA. Figure 1 shows the calibration curves air Kerma in PMMA versus intensity for X-ray beams of 120 and $135 \mathrm{kV}$.

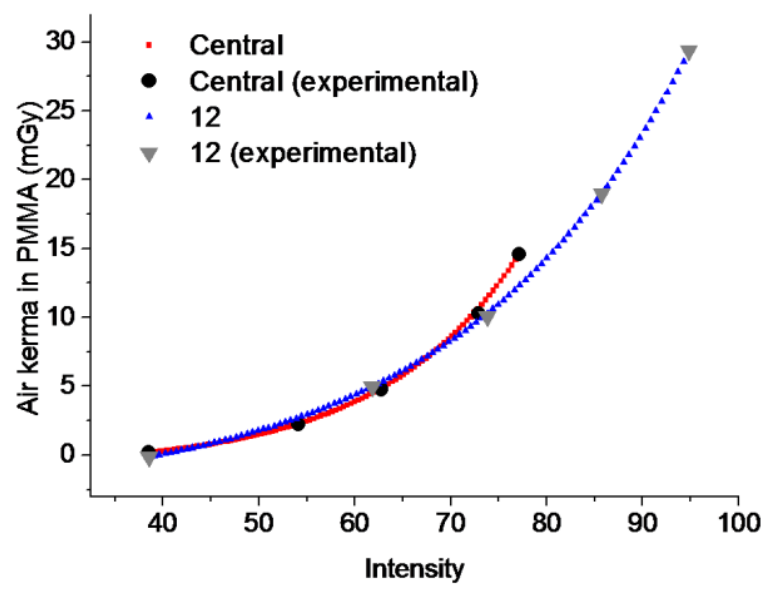

(a)

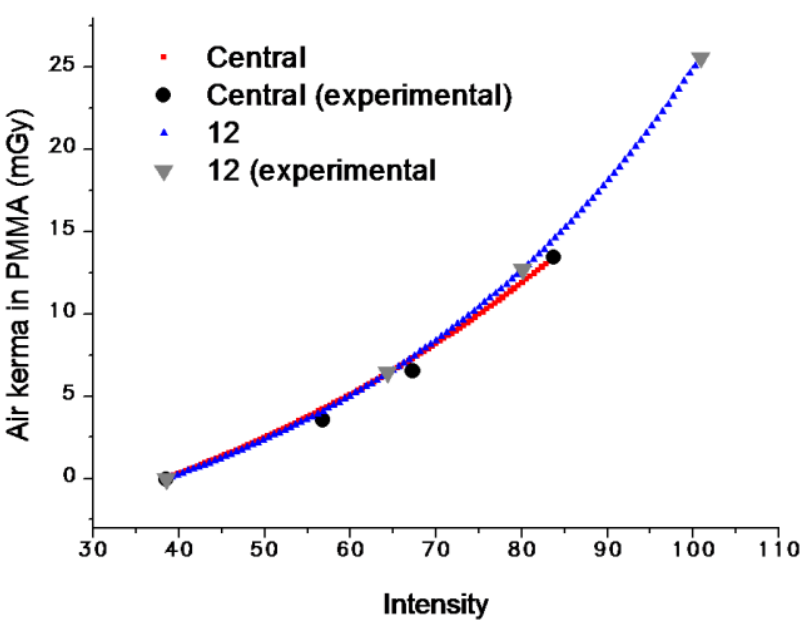

(b)

Figure 1: Calibration curves for calculates and experimental data at positions "Central" and “12” for the voltages of $120 \mathrm{kV}(\mathrm{a})$ and $135 \mathrm{kV}(\mathrm{b})$.

The difference in the darkening of the films when exposed allows observing the air Kerma in PMMA variations, and compare the differences in the X-ray beams. The responses obtained in the central and 12 positions had a considerable proximity to the voltage values with which the scans were performed. Although the $135 \mathrm{kV}$ voltage generates more deposition of energy compared to the $120 \mathrm{kV}$ voltage, the curves show similar responses. 
From the air Kerma in PMMA values recorded experimentally, calibration curves were obtained the curves that allow correlating the darkening of the irradiated strips with the corresponding air Kerma value. The mathematical models follow the expression:

$$
y=A * e^{(x / B)}-C
$$

Where $x$ corresponds to the greyscale intensity values of the film strip darkening and $y$ values of air Kerma in PMMA in mGy. The values found for $A, B$ and $C$ is presented in the table 1.

Table1. Values For the parameters $A, B$ and $C$ for calibration curves

\begin{tabular}{|c|c|c|c|c|}
\hline & \multicolumn{2}{|c|}{$120 \mathrm{kV}$} & \multicolumn{2}{|c|}{$135 \mathrm{kV}$} \\
\hline & CENTRAL & 12 & CENTRAL & 12 \\
\hline $\mathbf{A}$ & 0.078 & 0.614 & 2.022 & 3.077 \\
\hline B & 14.585 & 23.870 & 37.177 & 42.374 \\
\hline $\mathbf{C}$ & 0.926 & 3.210 & 5.759 & 7.661 \\
\hline
\end{tabular}

In the Tables 2 and 3 are compared to the values of Air Kerma in PMMA measured experimentally with the ionization chamber and the values of Air Kerma absorbed by films for each position calculated by equation 1 for the 120 and $135 \mathrm{kV}$ voltages. 
Table 2. Air Kerma in PMMA (mGy) measured and calculated values to $120 \mathrm{kV}$

\begin{tabular}{|c|c|c|c|c|c|}
\hline \multicolumn{3}{|c|}{ Central } & \multicolumn{3}{|c|}{12} \\
\hline Measured & Calculated & Error & Measured & Calculated & Error \\
\hline 0.000 & 0.172 & -0.172 & 0.000 & -0.125 & 0.125 \\
\hline 2.465 & 2.243 & 0.222 & 4.859 & 4.954 & -0.095 \\
\hline 4.931 & 4.780 & 0.151 & 9.718 & 10.085 & -0.367 \\
\hline 9.861 & 10.268 & -0.407 & 19.436 & 18.957 & 0.479 \\
\hline 14.792 & 14.589 & 0.203 & 29.153 & 29.380 & -0.227 \\
\hline
\end{tabular}

Table 3. Air Kerma in PMMA (mGy) measured and calculated values to $135 \mathrm{kV}$

\begin{tabular}{|c|c|c|c|c|c|}
\hline \multicolumn{3}{|c|}{ Central } & \multicolumn{3}{|c|}{12} \\
\hline Measured & Calculated & Error & Measured & Calculated & Error \\
\hline 0.000 & -0.059 & 0.059 & 0.000 & -0.022 & 0.022 \\
\hline 3.357 & 3.558 & -0.201 & 6.394 & 6.463 & -0.069 \\
\hline 6.713 & 6.540 & 0.173 & 12.789 & 12.730 & 0.059 \\
\hline 13.427 & 13.457 & -0.031 & 25.578 & 25.590 & -0.012 \\
\hline
\end{tabular}


The values of Air Kerma in PMMA calculated from using the equation 1 to intensity values recorded by radiochromic films have irrelevant errors when compared between the measured values and the ionization chamber values. The values with the lowest error correspond to values for 135 $\mathrm{kV}$ because the beam has a higher penetration in the matter.

\section{CONCLUSIONS}

The calibration curves for radiochromic films obtained allow realize punctual dosimetry and optimizing image acquisition protocols in Chest CT scans, using 120 and $135 \mathrm{kV}$ X-rays beams. These beams have different penetration characteristics.

When compared the curves obtained for 120 and $135 \mathrm{kV}$, there is an exponential response for both. However, considering the two voltage values, the $135 \mathrm{kV}$ beam generates higher air Kerma values in PMMA for the same charge value (mA.s).

The curves obtained for the central and 12 positions present similar behaviors for both voltages. The entrance of the X-ray beam occurs around the phantom $\left(360^{\circ}\right)$, so the central position receives a filtered beam by about $16 \mathrm{~cm}$ of PMMA, during all the irradiation time. The position 12 also receives a filtered beam, but the filtration varies from about 0.5 to $31 \mathrm{~cm}$ of PMMA, depending on the incidence angle of the beam.

\section{ACKNOWLEDGMENTS}

The researchers thank FAPEMIG and CAPES by support for the development of this study; likewise thank the Angus Dei clinical center, an institution with which partners and who allowed the development of experiments. 


\section{REFERENCES}

1. MOURÃO, A.P AND OLIVEIRA, F.A, Fundamentos de radiologia e imagem. São Paulo: Difusão Editora. First edition, 2009.

2. DOVALES, A. C. ET AL. Computed tomography in Brazil: frequency and pattern of usage among inpatients of the unified health system (sus). Revista Brasileira de Fisica Medica, v. 9, n. 1, p. 11-14, 2015.

3. DOVALES, A. C. ET AL. Patterns and trends of computed tomography usage in outpatients of the Brazilian public healthcare system, 2001-2011 Journal of Radiological Protection, IOP Publishing, v. 36, n. 3, p. 547, 2016.

4. GREFFIER, J. ET AL. Dose reduction with iterative reconstruction: optimization of CT protocols in clinical practice. Diagnostic and interventional imaging, Elsevier, v. 96, n. 5, p. 477-486, 2015.

5. GABUSI, M. ET AL. Radiation dose in chest CT: assessment of size-specific dose estimates based on water-equivalent correction. Physica Medica. Elsevier, v. 32, n. 2, p. 393-397, 2016.

6. GOMEZ, A. Estudo de dosimetria e qualidade de imagem em varreduras de tomografia computadorizada de cabeça utilizando objeto simulador. Dissertação (mestrado) Universidade Federal de Minas Gerais, Escola de Engenharia, 2017.

7. XU, X. G.; ECKERMAN, K. F. Handbook of anatomical models of radiation dosimetry. [S.1]: CRC press, 2009.

8. OLIVEIRA, A. C DE. ET AL. Construção de fantomas físicos para avaliação dosimétrica em planejamentos radioterapêuticos: $\mathrm{O}$ fantoma hydra.

9. ASHLAND INC. Gafchromic ${ }^{\mathrm{TM}}$ XR film. Gafchromic ${ }^{\mathrm{TM}}$ Radiology Films, [internet] Access Dec $10^{\text {th }}$ 2015. <http://www.ashland.com/products/gafchromic-radiology-films $>$.

10. RADCAL, C. ACCU-GOLD User Guide. [S.1.], 2015. 